\title{
Relações entre o nível de atividade física, autoestima e resiliência em pacientes com epilepsia
}

http://dx.doi.org/10.11606/1807-5509201900010039

Simone Thiemi KISHIMOTO*
Simone APPENZELLER
***
Paula Teixeira FERNANDES*

\section{Resumo}

A epilepsia é uma condição neurológica grave, caracterizada por crises recorrentes e espontâneas no cérebro. Devido à imprevisibilidade das crises e do preconceito existente na sociedade, alguns pacientes se isolam, prejudicando seu bem-estar físico, social e emocional. Métodos alternativos de tratamento, complementares aos medicamentosos são pesquisados com o objetivo de proporcionar uma melhor qualidade de vida a estes pacientes. Assim, pesquisas com exercício físico e atividade física mostram que a sua prática, pode proporcionar diversos benefícios na aptidão física e na saúde de pessoas saudáveis e também com diferentes tipos de doenças. Este estudo transversal tem como objetivo avaliar o nível de atividade física praticado pelos pacientes com epilepsia e comparar se os pacientes mais ativos possuem índices melhores de autoestima e resiliência. Foram entrevistados 80 pacientes com epilepsia de lobo temporal e utilizados os instrumentos: International Physical Activity Questionnaire (IPAQ), Escala de Autoestima de Rosenberg e Escala de Resiliência. Os resultados mostraram que $76,2 \%$ dos pacientes foram considerados ativos apresentando assim, melhores índices de autoestima $(p=0,001)$ e resiliência $(p=0,003)$, em comparação com grupo não ativo. Observamos uma relação significativa entre o número de crises e os níveis de autoestima e resiliência, com melhores niveis de autoestima $(p=0,046)$ e resiliência $(p=0,032)$ em pacientes considerados ativos com crises semanais, em relação ao grupo não ativo. A prática de atividade física pode contribuir como um importante método terapêutico complementar ao tratamento medicamentoso na epilepsia, promovendo a melhora da autoestima e resiliência nos pacientes, proporcionando deste modo uma melhor qualidade de vida.

Palavras-chave: Educação Física; Exercício; Doença Neurológica; Sintomas Psicológicos.

\section{Introdução}

A epilepsia é uma condição neurológica crônica, caracterizada por crises recorrentes e espontâneas no cérebro, que afeta cerca de 50 milhóes de pessoas no mundo todo, sem distinção de raça, sexo e regiōes ${ }^{1}$. Apesar de comum, pode prejudicar as funçóes cognitivas, emocionais e comportamentais, a capacidade de trabalho, funcionamento social, a estabilidade familiar e autoestima do paciente; influenciando assim sua qualidade de vida ${ }^{2}$.

As relaçôes familiares e sociais podem influenciar diretamente no modo como os pacientes lidam com a doença ${ }^{3}$, uma vez que a superproteção da família e o preconceito existente na sociedade afetam diretamente a autoestima destes pacientes ${ }^{4,5}$. Fatos que podem ser explicados devido as crises imprevisíveis, a incapacidade de controlar o próprio corpo, a dependência de medicamentos e de outras pessoas, que fazem com que os pacientes sintam-se sem controle sobre sua condição e sua vida ${ }^{6}$.

A autoestima é definida como um conjunto de características e atitudes internas do indivíduo, consistindo em um conjunto de pensamentos e sentimentos referentes a si mesmo ${ }^{7}$. Está diretamente relacionada à saúde mental e ao bem-estar psicológico. A baixa autoestima pode acarretar fenômenos mentais negativos, como sintomas depressivos e
*Faculdade de

Educação Física,

Universidade Estadual de Campinas,

Campinas, SP, Brasil.

${ }^{* *}$ Faculdade de

Ciências Médicas,

Universidade Estadual

de Campinas,

Campinas, SP, Brasil. 
suicídio $^{8}$. Um estudo ${ }^{9}$ realizado com adolescentes com epilepsia, apontou que as incertezas diante das condições da doença como a imprevisibilidade das crises e o desejo de fazer parte de um grupo diante do preconceito existente na sociedade, faz com que a autoestima e a autoconfiança sejam abaladas. Os pacientes começam a se achar diferentes das outras pessoas, limitando assim as oportunidades de crescimento acadêmico, profissional e pessoal ${ }^{9}$.

Assim como ocorre na autoestima, pesquisas sobre resiliência ${ }^{10-13}$ têm sido utilizadas cada vez mais para entender como os indivíduos se adaptam a doenças crônicas. BANDURA ${ }^{14}$ sugeriu que a resiliência pode ser vista como o resultado de um processo adaptativo de integração de novas metas no âmbito da saúde, diante das dificuldades.

Originado da Física, o termo resiliência é definido como um conjunto de processos sociais e intrapsíquicos que possibilita o desenvolvimento de uma vida sadia, mesmo vivendo em um ambiente nâo sadio ${ }^{15}$. É o processo de obtenção de resultados positivos inesperados em condiçóes adversas, e ocorre entre a pessoa e o meio em que se está inserido ${ }^{16}$.

Pacientes com doenças crônicas, constantemente precisam superar as adversidades causadas pela sua condição, sendo assim, ter bons índices de resiliência pode-se auxiliá-lo no controle do estresse, mantendo seu bem-estar psicológico e físico, proporcionando assim uma melhor qualidade de vida ${ }^{10}$. Portanto, se a resiliência pode afetar a qualidade de vida, maneiras de como o indivíduo consegue lidar com as instabilidades de sua condição, pode ser uma importante ferramenta para avaliar necessidades futuras. O conhecimento desta relação pode auxiliar na compreensão dos motivos pelos quais algumas pessoas conseguem manter níveis razoáveis de qualidade de vida, mesmo convivendo com uma doença crônica.

Assim como ocorre na resiliência, ter uma boa autoestima pode auxiliar na redução dos estímulos estressores da doença, principalmente no que se refere ao modo como o paciente lida com os preconceitos e todas as outras dificuldades ocasionadas pela sua condição $^{10}$. Uma autoestima positiva, poderia desta forma auxiliar no enfrentamento das adversidades, melhorando a adesão dos pacientes ao tratamento, atuando diretamente na sua qualidade de vida.

A Organização Mundial da Saúde ${ }^{17}$ aponta que a prática de atividades físicas, pode proporcionar diversos benefícios na aptidão física e na saúde de pessoas saudáveis e também com diferentes tipos de doenças. Porém os pacientes com epilepsia frequentemente são desencorajados e excluídos da participação em programas de atividade física, provocando deste modo uma queda na qualidade do seu bem-estar físico, emocional, social e consequentemente redução da autoestima ${ }^{18,19}$.

Neste sentido, se a atividade física pode atuar de maneira positiva na saúde e bem-estar de pessoas com doenças crônicas e saudáveis, qual seriam seus efeitos nos aspectos psicológicos em pacientes com epilepsia? Assim, esta pesquisa teve como objetivo avaliar a relação entre os níveis de atividade física praticados pelos pacientes com epilepsia de lobo temporal, e os índices de autoestima e resiliência apresentados pelos mesmos.

\section{Método}

Foram entrevistados 80 pacientes com epilepsia de lobo temporal, do Ambulatório de Neurologia HC/Unicamp, com idade entre 18 a 60 anos (42,5 $\pm 10,0$ anos), sendo 49 mulheres e 31 homens.

Para a seleção dos voluntários, todos deveriam estar em tratamento com medicação antiepiléptica, além de não terem sido submetidos à cirurgia para epilepsia e nem possuir transtornos mentais graves (esquizofrenia, surtos psicóticos, depressão grave, entre outros). Os dados foram obtidos através da revisão de prontuários e acompanhamento durante o retorno médico. Foram excluídos 8 pacientes que não se enquadravam nos quesitos necessários para a pesquisa.

Os participantes foram entrevistados em uma sala localizada no Ambulatório de Neurologia do Hospital das Clínicas da Unicamp, no mesmo dia do retorno com o médico. Todos os voluntários que aceitaram participar da pesquisa, assinaram o Termo de Consentimento Livre e Esclarecido (TCLE), informando os possíveis riscos e benefícios do estudo, aprovado pelo Comitê de Ética em pesquisa em seres humanos da Plataforma Brasil, com o processo de número 488.402 . 


\section{Descrição dos instrumentos}

Para essa pesquisa foram utilizados 4 instrumentos:

1) Ficha de identificação contendo: nome, idade, sexo, escolaridade, emprego e dados sobre a condição (início, tipo de crise, medicação, frequência e data da última crise).

2) Questionário Internacional de atividade física (International Physical Activity Questionnaire) em sua versão curta (IPAQ) validado para a população brasileira por MATSUdo et al..$^{20}$ : utilizado para a triagem de atividade física, foi aplicada a versão reduzida, composta de 8 perguntas relacionadas ao tempo que o indivíduo despende fazendo atividade física na última semana. As perguntas incluem atividades no trabalho, para ir de um lugar ao outro, por lazer, por esporte, por exercício ou como parte de suas atividades em casa. O questionário avalia os indivíduos em 5 níveis de atividade física: muito ativo, ativo, irregularmente ativo A, irregularmente ativo $B$ e sedentário. Para viabilizar a análise estatística, reduzimos esses 5 grupos para 2 grupos: Grupo Ativo (muito ativo e ativo) e Grupo Não Ativo (irregularmente ativo $\mathrm{A}$, irregularmente ativo B e sedentário). Os sujeitos foram divididos em ativos e não ativos segundo as classificaçóes do questionário IPAQ (versão curta) ${ }^{20}$. Os participantes foram divididos em 3 faixas etárias, segundo a teoria do desenvolvimento humano: Grupo $A=18-20$ anos (2 pacientes), Grupo $B=21 \sim 40$ anos (25 pacientes) e Grupo $\mathrm{C}=41-60$ anos (53 pacientes).

3) Escala de Autoestima Rosenberg (Rosenberg Self-Esteem Scale $)^{21,22,}$, validado para a população brasileira por Dini et al. ${ }^{23}$ : avalia a atitude e o sentimento positivo ou negativo por si mesmo. Abrange questôes de satisfação pessoal, autodepreciação, percepção de qualidades, competência, orgulho por si, autovalorização, respeito e sentimento de fracasso. Composta por uma escala do tipo Likerd, contendo 10 questóes fechadas, com as seguintes opçóes de resposta: concordo totalmente, concordo, discordo e discordo totalmente, sendo que cada item de resposta varia de 0 a 3 pontos. $\mathrm{O}$ escore pode variar de 0 a 30 pontos. Quanto menor o escore, melhor o nível de autoestima.

4) Escala de Resiliência (Resilience Scale) ${ }^{24}$, validado para a população brasileira por PeSCE et al. ${ }^{15}$ : desenvolvida para medir níveis de adaptação psicossocial positiva em face aos eventos de vida importantes. Possui 25 itens descritos de forma positiva, variando de 1 (discordo totalmente) a 7 (concordo totalmente), sendo que seu escore pode variar de 25 a 175 pontos, com valores altos, indicando elevada resiliência.

\section{Análise dos dados}

A análise estatística foi realizada através de testes apropriados para cada tipo de variável, a fim de comparar os resultados dos questionários aplicados. Utilizamos o software SYSTAT ${ }^{\mathrm{TM} 15}$ para analisar diferenças de variáveis contínuas entre grupos. Foi realizado a correçáo Bonferroni. O teste utilizado para detectar possíveis diferenças entre os grupos foi o Anova, e foi utilizado o teste ' $\mathrm{t}$ ' de Student para amostras dependentes para comparar resultados. Realizamos correlações de Pearson entre variáveis contínuas. O nível de significância adotado foi de $\mathrm{p}<0,05$.

\section{Resultados}

No total, foram entrevistados 80 pacientes com epilepsia de lobo temporal, com o objetivo de verificar o nível de atividades físicas praticados e a sua relação com a autoestima e resiliência.

Com relação às drogas antiepilépticas, observamos que $15(18,7 \%)$ pacientes tomavam somente 1 tipo de medicamento e $65(81,2 \%)$ pacientes tomavam 2 ou mais medicamentos, caracterizando assim a politerapia. Os medicamentos administrados são mostrados na TABELA 1.
Ao comparar a prática de atividades físicas, Grupo Ativo e Grupo Não ativo nas diferentes faixas etárias, observamos que o grupo $\mathrm{C}$ (41 -60 anos) tem pacientes mais ativos que os grupos $A(18-20$ anos) e B (21 40 anos) com p = 0,025 (TABELA 2).

A autoestima de pacientes ativos $(6,68)$ mostrou-se menor em comparação ao grupo não ativo $(11,68)$, com análise estatística significativa $(\mathrm{p}=0,001)^{\text {Mann-Whitney }}$. Salientamos que quanto menor o escore, melhor a autoestima (FIGURA 1). Nível de significância adotado foi de $\mathrm{p}<0,05$. 
TABELA 1 - Drogas antiepilépticas administradas pelos pacientes

\begin{tabular}{lcc}
\hline Drogas antiepilépticas & Pacientes ativos (\%) & Pacientes não ativos (\%) \\
\hline Carbamazepina & $42(68,8 \%)$ & $14(73,3 \%)$ \\
Clobazan & $29(47,5 \%)$ & $11(57,8 \%)$ \\
Valproato & $12(19,6 \%)$ & $2(10,5 \%)$ \\
Lamotrigina & $11(18 \%)$ & $4(21 \%)$ \\
Topiramato & $7(11,4 \%)$ & $5(26,3 \%)$ \\
Fenobarbital & $2(3,2 \%)$ & $1(5,2 \%)$ \\
Oxicarbamazepina & $2(3,2 \%)$ & $0(0 \%)$ \\
\hline
\end{tabular}

Autoestima: escore 0-30; Resiliência: escore 25-175; Grupo Ativo: muito ativo e ativo, segundo IPAQ; Grupo não ativo: irregularmente ativo A, irregularmente ativo $B$ e sedentário, segundo IPAQ. Grupos A, B e C divididos por idade segundo a Teoria do Desenvolvimento Humano ${ }^{25}$. Nível de significância adotado $\mathrm{p}<0,05$. ${ }^{\text {TTeste }}$ Quiquadrado.

TABELA 2 - Dados dos pacientes entrevistados

\begin{tabular}{lccccc}
\hline & Feminino & Masculino & $\begin{array}{c}\text { Grupo A } \\
\mathbf{1 8 - 2 0} \text { anos }\end{array}$ & $\begin{array}{c}\text { Grupo B } \\
\mathbf{2 1 - 4 0} \text { anos }\end{array}$ & $\begin{array}{c}\text { Grupo C } \\
\mathbf{4 1 - 6 0} \text { anos }\end{array}$ \\
\hline Sujeitos & 49 & 31 & 2 & 25 & 53 \\
Autoestima (média escore) & 8,48 & 6,9 & 6 & 7,84 & 7,96 \\
Resiliência (média escore) & 139,14 & 138,45 & 145,5 & 137,28 & 139,37 \\
Grupo Ativo & 36 & 25 & 0 & 18 & 43 \\
n (\%) & $(73,4 \%)$ & $(80,6 \%)$ & $(0 \%)$ & $(72 \%)$ & $(81,1 \%)$ \\
Grupo náo ativo & 13 & 6 & 2 & 7 & 10 \\
n (\%) & $(26,6 \%)$ & $(19,3 \%)$ & $(100 \%)$ & $(28 \%)$ & $(18,9 \%)$ \\
p & & & $\mathrm{p}=0,187^{\mathrm{a}}$ & $\mathrm{p}=0,203^{\mathrm{a}}$ & $\mathbf{p}=\mathbf{0 , 0 2 5}$ \\
\hline
\end{tabular}

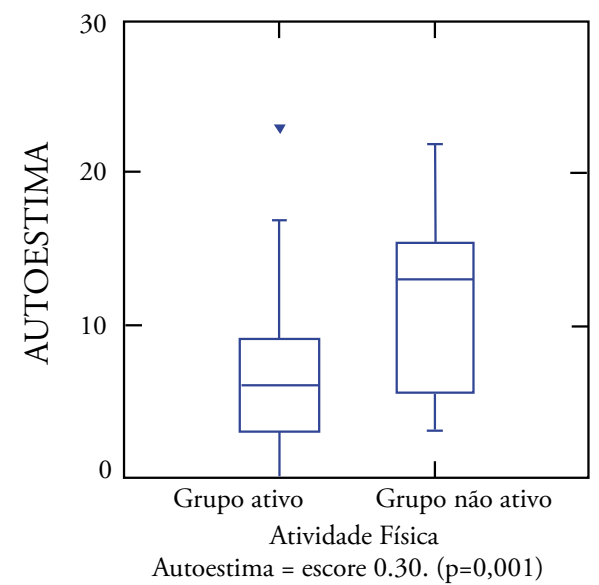

FIGURA 1 - Relação entre autoestima e nível de atividade física, Grupo ativo e Grupo não ativo. Autoestima: escore $0-30 ; p=0,001$.

Observamos que quanto menor a frequência de crises, menor a média de escore de autoestima, o que indica melhor autoestima em pacientes com menos crises. Ao comparar a média de autoestima em pacientes ativos e não ativos, observamos que o grupo ativo apresenta médias de escores menores de autoestima (melhor autoestima) em relação ao grupo não ativo, com análise estatística significativa nos pacientes com crises semanais $(\mathrm{p}=0,046)$ (TABELA 3$)$.
A análise dos resultados mostrou diferença significativa entre o Grupo Ativo e Grupo Não Ativo, quando comparado com os escores de resiliência. A resiliência de pacientes ativos $(141,95)$ mostrou-se maior em comparação ao grupo não ativo $(129,00)(\mathrm{p}=0,003)^{\text {Mann-Whinney }}$. Quanto maior o escore, melhor o índice de resiliência. Nível de significância adotado foi de $\mathrm{p}<0,05$ (FIGURA 2). 
Ao comparar a frequência de crises e a média dos escores de resiliência, observamos que quanto maior o número de crises, menor a média de escore de resiliência, o que indica uma menor percepção de resiliência em pacientes com mais crises. Ao comparar a média de resiliência em pacientes ativos e não ativos, observamos que o grupo ativo apresenta escores maiores de resiliência (melhor resiliência) em relaçáo ao grupo não ativo, com análise estatística significativa para o grupo com crises semanais $(p=0,032)$, e mensais $(p=0,021)$ (TABELA 4).

TABELA 3 - Relação entre o número de crises e a média de autoestima

\begin{tabular}{lcccc}
\hline Crise & Semanal & Quinzenal & Mensal & Anual \\
\hline Ativo (pacientes) & 17 & 10 & 18 & 16 \\
Não Ativo (pacientes) & 11 & 4 & 2 & 2 \\
Total (pacientes) & 28 & 14 & 20 & 18 \\
Autoestima (grupo ativo) (média \pm DP) & $9,29 \pm 6,3$ & $8 \pm 5,0$ & $5,22 \pm 2,1$ & $4,75 \pm 2,8$ \\
Autoestima (grupo náo ativo) (média \pm DP) & $14,18 \pm 11,0$ & $10,25 \pm 6,6$ & $9 \pm 5,7$ & $3,5 \pm 1,6$ \\
Autoestima (total) (média \pm DP) & $11,21 \pm 7,4$ & $8,6 \pm 4,3$ & $5,6 \pm 2,2$ & $4,61 \pm 1,4$ \\
$\mathbf{p}$ & $\mathbf{p = \mathbf { 0 , 0 4 6 } ^ { \mathrm { b } }}$ & $\mathrm{p}=0,634^{\mathrm{b}}$ & $\mathrm{p}=0,514^{\mathrm{b}}$ & $\mathrm{p}=0,261^{\mathrm{b}}$ \\
\hline
\end{tabular}

${ }^{\mathrm{b}}$ Teste Kruscal-Wallis. Nível de significância adotado $p<0,05$.

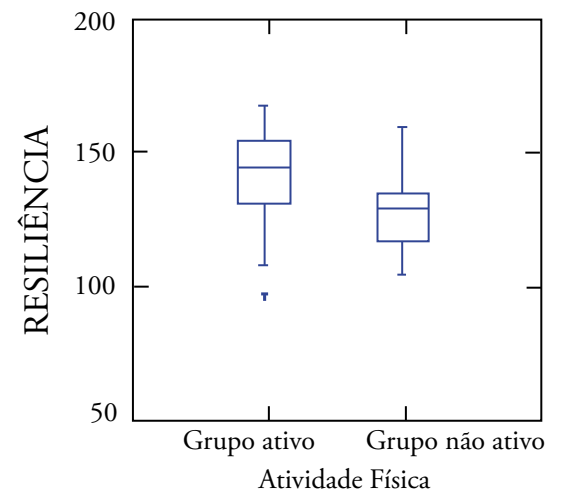

FIGURA 2 - Relação entre resiliência e nível de atividade física, Grupo Ativo e Grupo não ativo. Resiliência: escore 25-175; $\mathrm{p}=0,003$.

TABELA 4 - Relação entre o número de crises e a média de resiliência

\begin{tabular}{lcccc}
\hline Crise & Semanal & Quinzenal & Mensal & Anual \\
\hline $\begin{array}{l}\text { Ativo (pacientes) } \\
\text { Náo Ativo (pacientes) }\end{array}$ & 17 & 10 & 18 & 16 \\
Total (pacientes) & 11 & 4 & 2 & 2 \\
$\begin{array}{l}\text { Resiliência (grupo ativo) } \\
\text { (média } \pm \text { DP) }\end{array}$ & 28 & 14 & 20 & 18 \\
$\begin{array}{l}\text { Resiliência (grupo náo ativo) } \\
\text { (média } \pm \text { DP) }\end{array}$ & $136,1 \pm 103,0$ & $136,8 \pm 98,1$ & $146,8 \pm 112,5$ & $145,8 \pm 86,2$ \\
$\begin{array}{l}\text { Resiliência (total) } \\
\text { (média } \pm \text { DP) }\end{array}$ & $125,9 \pm 93,8$ & $130,0 \pm 103,3$ & $130,5 \pm 96,5$ & $142,5 \pm 79,6$ \\
$\mathbf{p}$ & $132,1 \pm 106,4$ & $134,8 \pm 102,0$ & $145,2 \pm 114,0$ & $145,5 \pm 99,1$ \\
\hline
\end{tabular}

${ }^{\mathrm{b}}$ Teste Kruscal-Wallis. Nível de significância adotado $p<0,05$. 


\section{Discussão}

Os resultados apresentados neste estudo mostraram que pacientes com epilepsia que praticam algum tipo de atividade física, apresentam melhores escores de autoestima $(\mathrm{p}=0,001)$ e resiliência $(\mathrm{p}=0,003)$ em relação ao grupo náo ativo. UNGAR ${ }^{12}$ salienta que o modo como o paciente lida com a doença e suas concepçóes de normalidade sobre o modo de vida, são fundamentais para o desenvolvimento da resiliência, uma vez que; em pessoas com doenças crônicas isso ocorre dentro de um contexto cultural e social.

Identificamos nesta pesquisa que dos 80 pacientes entrevistados, 55\% (44 participantes) não trabalhavam, porque estavam desempregados ou eram aposentados, e 45\% (36 voluntários) trabalhavam regularmente ou exerciam algum tipo de atividade remunerada fora de casa. Contrapondo os dados apresentados neste estudo, Wo et al. ${ }^{26}$ identificaram que dos 146 pacientes com epilepsia avaliados, a maior parte $64 \%$ estavam empregados, observou-se que este grupo apresentou maior motivação, autoestima, suporte social e menor estigma em comparaçáo ao grupo que estava sem emprego. Os autores ${ }^{26}$ indicam que fatores ligados ao tipo de epilepsia, nível de escolaridade e aspectos psicológicos, afetam diretamente a empregabilidade destes pacientes.

Ao dividir os pacientes pela faixa etária, observamos que os pacientes do grupo C (41 -60 anos) são mais ativos em relação aos outros dois grupos (A e B). Uma possível explicação é o fato da maioria dos pacientes desta faixa etária já serem aposentados por invalidez, sendo assim possuem mais horas livres para a prática de atividades físicas.

Neste estudo, identificamos que a maioria dos participantes $(76,2 \%)$ considerada ativa, as principais atividades físicas praticadas pelos pacientes eram: deslocamento de um local para outro, atividades de trabalho, serviços domésticos e caminhada. Um estudo de Arida et al. ${ }^{27}$ contrapóes os dados que identificamos neste estudo, pois dos 100 pacientes avaliados pelos autores somente $15 \%$ praticavam atividades físicas. Porém, salientamos que no estudo de Arida et al. $^{27}$ a seleção foi realizada através de um questionário pré-elaborado e não incluía as atividades de deslocamento, no trabalho e domésticas; englobando somente os exercícios físicos e atividades de lazer.

Gordon e Dooley ${ }^{28}$ também mostraram a caminhada como a principal atividade de lazer praticada pelos pacientes com epilepsia, fato este que corrobora com o apresentado nesta pesquisa. Os autores apontaram que muitos pacientes são impedidos de dirigir por causa dos riscos de acidentes e também evitam andar de bicicleta por causa do medo de sofrer quedas e lesóes ${ }^{28}$. BRNA et al. ${ }^{29}$ avaliaram adolescentes com epilepsia e seus pais, foram identificados que os jovens apresentam diversas restriçóes com relação a prática de atividades físicas e de vida diárias, devido ao medo e ao risco de acidentes, além disso os pais mostraram-se bastante cautelosos com relação as atividades praticadas pelos filhos ${ }^{29}$.

KwONG et al..$^{30}$ avaliaram a autoestima de 140 adolescentes com epilepsia e de 50 crianças com asma, identificando menor autoestima nos pacientes com epilepsia. Os autores ${ }^{28}$ acreditam que o resultado deve-se a natureza estigmatizante da doença, causada pela superproteção dos pais e restriçóes de atividades. Salienta-se ainda que a baixa autoestima em adolescentes com epilepsia pode acarretar importantes implicações no funcionamento psicológico, principalmente nesta fase que é cercada de grandes mudanças físicas, fisiológicas e sociais ${ }^{30}$.

Em relação ao número de crises, observamos uma tendência de melhores índices de autoestima e resiliência em pacientes com menor número de crises e também nos pacientes considerados ativos. Porém salientamos que os pacientes com crises semanais considerados ativos, apresentaram melhores índices de autoestima $(\mathrm{p}=0,046) \mathrm{e}$ resiliência $(p=0,032)$ em comparação ao grupo não ativo. Destacamos assim, a importância da prática de atividades físicas principalmente em pacientes com um número maior de crises.

Estes dados, corroboram com o estudo de Ring et al. ${ }^{13}$ que apontaram que muitos pacientes mesmo com um número elevado de crises, apresentam boa qualidade de vida. Os autores ${ }^{13}$ acreditam que este fato ocorre, devido a influências de aspectos pessoais (espírito de luta, entusiasmo pela vida, modos de como suprir determinadas perdas causadas pela doença e aceitação da sua condição) e ambientais (apoio 
da família e amigos), além de suporte e amparo dentro do ambiente de trabalho.

SAWANCHAREON et al. ${ }^{31}$ apresentaram que o uso prolongado de drogas antiepilépticas e a frequência de crises podem afetar negativamente a autoestima nos pacientes com epilepsia. TAYLOR et al. ${ }^{11}$ mostraram que o número de crises está relacionado diretamente à qualidade de vida dos pacientes com epilepsia. SAlgado e Souza ${ }^{32}$ destacam em seu estudo, que o medo de ter crises influencia diretamente à qualidade de vida dos pacientes, mais do que a própria frequência de crises em si, fato que corrobora com os resultados deste estudo, uma vez que pacientes com maior número de crises considerados ativos, apresentaram melhores índices de autoestima e resiliência.

Volpato et al..$^{33}$, avaliaram o nível de atividade física e qualidade de vida em pacientes com epilepsia, apresentando uma tendência de menor medo de crises nos pacientes considerados ativos, corroborando com os dados deste estudo. Os autores $^{33}$ sugerem que o grupo ativo apresenta uma maior independência e enfrenta melhor sua condição, o que faz com que com eles realizem atividades cotidianas como trabalho, serviços domésticos e atividades de lazer.

As influências fisiológicas da prática de atividades físicas na epilepsia foram identificadas por van Linschoten et al. ${ }^{34}$. Os autores ${ }^{34}$ mostraram que durante o exercício, ocorre no nosso organismo a liberaçáo de beta-endorfina, que atua como anticonvulsivante, diminuído a atividade epiléptica. Além disso, este hormônio é responsável pela sensação de bem-estar, diminuindo o estresse e tensão; que podem ser fatores desencadeadores de crises ${ }^{35}$.

Nos anos seguintes, outros estudos ${ }^{36-39}$ envolvendo a atividade física na epilepsia foram realizados. NAKken et al. ${ }^{36}$ elaboraram um programa de atividades físicas com 26 crianças (média de idade de 10 anos) em ciclo ergômetro até a exaustão, destas 20 crianças apresentaram diminuição das descargas epilépticas durante a atividade. MCLAURIN ${ }^{37}$, realizaram um programa de atividades físicas com 23 pacientes durante 12 semanas e viram melhora na qualidade de vida no grupo que praticou atividades físicas.

RAUCHENZAUNER et al. ${ }^{38}$ (2017) avaliaram 107 crianças com epilepsia e identificou que a qualidade de vida está diretamente associada a aptidão física. Goodwin e STAPLES ${ }^{39}$ apontaram que a prática de atividades físicas por pacientes com doenças crônicas aumenta a oportunidade de socialização, permitindo a troca de experiências comuns entre eles possibilitando deste modo a aceitaçáo e inclusão social e consequentemente o aumento da autoestima.

Identificamos alguns estudos ${ }^{29,40-43}$ que abordam a resiliência na epilepsia, porém nenhum incluía a atividade física. WAGNER et al. ${ }^{40}$ avaliaram 9 jovens (10 a 15 anos) com epilepsia, onde foi aplicado um programa para o aprimoramento das habilidades de enfrentamento, autoeficácia e autogestão para promoção da resiliência de pacientes e seus cuidadores. Neste estudo ${ }^{40}$, foram identificados que os pais avaliaram melhorias significativas nas habilidades de enfrentamento após a intervenção, e as crianças avaliaram melhorias significativas na autoeficácia para o gerenciamento da doença. Os autores ${ }^{40}$ salientam a importâncias de estratégias que visem o desenvolvimento da resiliência nestes pacientes, para melhor enfrentamento das adversidades da doença.

Ring et al. ${ }^{29}$ citam que fatores pessoais, sociais, ambientais e o número de crises influenciam diretamente a qualidade de vida e a resiliência nos pacientes com epilepsia. Para a promoção de uma boa resiliência, os pacientes precisam encontrar recursos ambientais para que possa enfrentar as adversidades da doença, além de suporte da família, trabalho, psicológico e assistência a um tratamento adequado ${ }^{29}$.

Os cuidados com a saúde para que o paciente possa enfrentar resilientemente as adversidades provocadas pela doença são fundamentais. Na epilepsia, a resiliência pode ser apresentada como um fator de proteção contra sintomas depressivos, além de fortalecimento e superação de situações adversas como a frequência de crises, problemas sociais e psicológicos ${ }^{41,42}$. Bons índices de resiliência podem resultar em um maior autodomínio e menor percepção de estigma deste paciente, levando ao aumento da qualidade de vida ${ }^{43}$.

Assim como ocorre na resiliência, índices melhores de autoestima podem proporcionar diversos benefícios aos pacientes com epilepsia, como aumento da adesáo ao tratamento, reduçáo do preconceito, redução dos problemas de comportamento, melhora da saúde mental e bemestar psicológico, consequentemente melhoria da qualidade de vida. 
Apesar das limitaçóes deste estudo, devido á ausência de grupo controle, possibilitando possíveis comparações dos resultados, concluímos que estratégias de intervenção como a prática de atividade física, podem atuar como um importante método terapêutico complementar ao tratamento medicamentoso para a melhora da autoestima e resiliência em pacientes com epilepsia, fatores fundamentais para uma melhor qualidade de vida.

\section{Abstract}

Relationship between physical activity level, self-esteem and resilience in patients with epilepsy

Epilepsy is a serious neurological condition characterized by recurrent, spontaneous seizures in the brain. Due to the unpredictability of crises and the existing prejudices in society, some patients are isolated, damaging their physical well-being, social and emotional. Complementary treatment methods allied to the drug are searched, with the aim of providing a better quality of life for these patients. Thus, research on exercise and physical activity show that their practice, can provide many benefits in physical fitness and health of healthy people and also with different types of diseases. This study aims to assess the degree of physical activity practiced by patients with epilepsy and compare it the most active patients have better levels of self-esteem and resilience. They interviewed 80 patients with temporal lobe epilepsy and used instruments: International Physical Activity Questionnaire (IPAO), Self-Esteem Scale and Resilience Scale. The results show that $76.2 \%$ of the patients were considered active, presenting better levels of self-esteem indexes $(p=0.001)$ and resilience $(p=0.003)$ compared with non-active groups. We observed a significant relationship between the number of seizures and the levels of self-esteem and resilience, with better levels of self-esteem $(p=0.046)$ and resilience $(p=0.032)$ in patients considered active with weekly seizures compared to the non-active group. The practice of physical activity may contribute as an important therapeutic method complementary to drug treatment in epilepsy, promoting the improvement of self-esteem and resilience in patients with epilepsy, thus providing a better quality of life.

KeYwords: Physical Education; Exercise; Neurological Disease; Psychological Symptoms.

\section{Agradecimentos}

Agradecemos a Fundação de Amparo à Pesquisa do Estado de São Paulo (FAPESP), pelo auxílio concedido para a realização desta pesquisa.

\section{Referência}

1. World Health Organization. Fact sheet n. 999: Epilepsy [Internet]. Geneva: WHO; 2015 [citado 25 jul 2019]. Disponível em: http://who.int/mediacentre/factsheets/fs999/en/print.html.

2. Guerreiro CA, Guerreiro MM. O paciente otimamente controlado. São Paulo: Lemos; 1999.

3. Aydin K, Yildiz H. Teachers' perceptions in central Turkey concerning epilepsy and asthma and the short-term effect of a brief education on the perception of epilepsy. Epilepsy Behav. 2007;10(2):286-90.

4. Austin JK, Huster GA, Dunn D, Risinger MW. Adolescents with active or inactive epilepsy or asthma: a comparison of quality of life. Epilepsia. 1996;37(12):1228-38.

5. McEwan MJ, Espie CA, Metcalfe J. A systematic review of the contribution of qualitative research to the study of quality of life in children and adolescents with epilepsy. Seizure. 2004;13(1):3-14.

6. Amir M, Roziner I, Knoll A, Neufeld MY. Self-efficacy and social support as mediators in the relation between disease severity and quality of life in patients with epilepsy. Epilepsia. 1999;40(2):216-24. 
7. Siqueira NF, Guerreiro MM, Souza EAP. Self-esteem, social support perception and seizure controllability perception in adolescents with epilepsy. Arq Neuropsiquiatr. 2011;69(5):770-4.

8. Andrade D, Angerami ELS. A autoestima em adolescentes com e sem fissuras de lábio e/ou de palato. Rev Latino-Am Enfermagem. 2001;9(6):37-41.

9. Fernandes PT, Li LM. Percepção de estigma na epilepsia. J Epilepsy Clin Neurophysiol. 2006;12(4):207-18.

10. Yi JP, Vitaliano PP, Smith RE, Yi JC, Weinger K. The role of resilience on psychological adjustment and physical health in patients with diabetes. Br J Health Psychol. 2008;13(Pt 2):311-25.

11. Taylor J, Jacoby A, Baker AG, Ring A, Whitehead M. Factors predictive of resilience and vulnerability in new-onset epilepsy. Epilepsia. 2011;52(3):610-18.

12. Ungar M. Resilience, trauma, context and culture. Trauma Violence Abuse. 2013;14(3):255-66.

13. Ring A, Jacoby A, Baker GA, Marson A, Whitehead MM. Does the concept of resilience contribute to understanding good quality of life in the context of epilepsy? Epilepsy Behav. 2016;56:153-64.

14. Bandura A. The primacy of self-regulation in health promotion. Appl Psychol. 2005;54(2): 245-54.

15. Pesce RP, Assis SG, Avanci JQ, Santos NC, Malaquias JV, Carvalhaes R. Adaptação transcultural, confiabilidade e validade da escala de resiliência. Cad Saúde Pública. 2005;21(2): 436-48.

16. Canvin K, Marttila A, Burstrom B, Whitehead M. Tales of the unexpected? Hidden resilience in poor households in Britain. Soc Sci Med. 2009;69(2):238-45.

17. Organização Mundial da Saúde. Epilepsy [Internet]. Geneva: OMS; 2017 [citado 12 set 2017]. Disponível em: http://www. who.int/mediacentre/factsheets/fs999/es/.

18. Arida RM, Cavalheiro EA, Silva AC, Scorza FA. Physical activity and epilepsy: proven and predicted benefits. Sports Med. 2008;38(7):607-15.

19. Vieira D, Scorza FA, Silva AC, et al. Efeitos benéficos do exercício físico nas epilepsias: o judô faz parte deste contexto? J Epilepsy Clin Neurophysiol. 2007;13(3):131-6.

20. Matsudo S, Araújo T, Matsudo V, et al. Questionário Internacional de Atividade Física (IPAQ): estudo de validade e reprodutibilidade no Brasil. Ativ Fís Saúde. 2001;6(2):6-18.

21. Rosenberg M. Society and the adolescent self-image. Princeton: Princeton University Press; 1989.

22. Rosenberg M, Schooler C, Schoenbach C, Rosenberg F. Global self-esteem and specific self-esteem: different concepts, different outcomes. Am Sociol Rev. 1995;60(1):141-56.

23. Dini GM, Quaresma MR, Ferreira LM. Adaptação cultural e validação da versão brasileira da Escala de Autoestima de Rosenberg. Rev Bras Cir Plást. 2004;19(1):41-52.

24. Wagnild GM, Young HM. Development and psychometric evaluation of resilience scale. J Nurs Meas. 1993;1:165-78.

25. Papalia DE, Olds WS, Feldman RD. Desenvolvimento humano. 10a ed. Porto Alegre: Artmed; 2007.

26. Wo MC, Lim KS, Choo WY, Tan CT. Factors affecting the employability in people with epilepsy. Epilepsy Res. 2016;128:6-11.

27. Arida RM, Scorza FA, Albuquerque M, Cysneiros RM, Oliveira RJ, Cavalheiro EA. Evaluation of physical exercise habits in Brazilian patients with epilepsy. Epilepsy Behav. 2003;4(5):507-10.

28. Gordon E, Dooley JM. Epilepsy and activity: population-based study. Epilepsia. 2010;51(11):2254-9.

29. Brna PM, Gordon KE, Woolridge E, Dooley JM, Wood E. Perceived need for restrictions on activity for children with epilepsy. Epilepsy Behav. 2017;73:236-9

30. Kwong KL, Lam D, Tsui S, et al. Self-esteem in adolescents with epilepsy: Psychosocial and seizure-related correlates. Epilepsy Behav. 2016;63:118-22.

31. Sawanchareon K, Pranboon S, Tiamkao S, Sawanyawisuth K. Moving the self-esteem of people with epilepsy by supportive group: a clinical trial. J Caring Sci. 2013;2(4):329-35.

32. Salgado PCB, Souza EAP. Variáveis psicológicas envolvidas na qualidade de vida de portadores de epilepsia. Estud Psicol. 2003;8(1):165-8.

33. Volpato N, Cendes F, Yasuda LY. Relação entre qualidade de vida e atividade física em pacientes com epilepsia de lobo temporal refratária. J Epilepsy Clin Neurophysiol. 2011;17(4):127-32.

34. van Linschoten R, Backx FJG, Mulder OGM, Meinardi H. Epilepsy and sports. Sports Med. 1990;10(1):9-19.

35. Cooper K. O programa aeróbico para o bem estar total. Rio de Janeiro: Nórdica; 1982.

36. Nakken KO, Loyning A, Loyning T, Gloersen G, Larsson PG. Does physical exercise influence the occurrence of epileptiform EEG discharges in children? Epilepsia. 1997;38(3):279-84.

37. Mclaurin RL. Epilepsy and contact sports: factors contraindicanting 20. participation. In: Harris P, Mawdsley C. Epilepsy. Edinburgh: Churchill Livingstone; 1974. p. 301-5. 
38. Rauchenzauner M, Hagn C, Walch R, et al. Quality of life and fitness in children and adolescents with epilepsy (EpiFit). Neuropediatrics. 2017;48:161-5.

39. Goodwin DL, Staples K. The meaning of summer camp experiences to youths with disabilities. Adapt Phys Activ Q. 2005;22(2):160-78.

40. Wagner JL, Smith G, Ferguson P, van Bakergem K, Hrisko S. Pilot study of an integrated cognitive-behavioral and selfmanagement intervention for youth with epilepsy and caregivers: Coping Openly and Personally with Epilepsy (COPE). Epilepsy Behav. 2010;18:280-5.

41. Jacoby A, Baker G. Quality of life trajectories in epilepsy: a review of the literature. Epilepsy Behav. 2008;12(4):557-71.

42. Austin JK, Perkins SM, Johnson CS, et al. Self-esteem and symptoms of depression in children with seizures: relationships with neuropsychological functioning and family variables over time. Epilepsia. 2010;51:2074-83.

43. Reisinger EL, Dilorio C. Individual, seizure-related, and psychosocial predictors of depressive symptoms among people with epilepsy over six months. Epilepsy Behav. 2009;15(2):196-201.

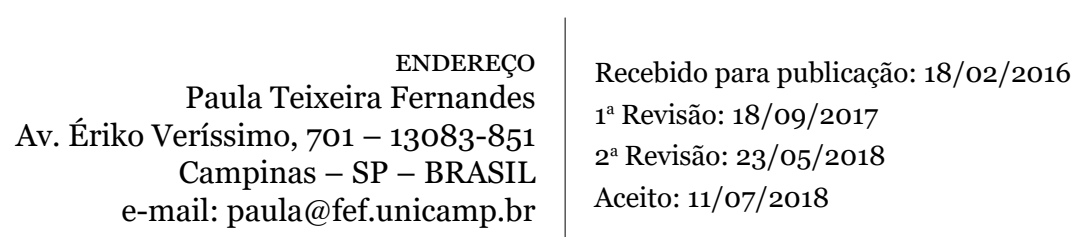

